\title{
Pangenome-wide and molecular evolution analyses of the Pseudomonas aeruginosa species
}

Jeanneth Mosquera-Rendón ${ }^{1,2}$, Ana M. Rada-Bravo ${ }^{3,4}$, Sonia Cárdenas-Brito ${ }^{1}$, Mauricio Corredor ${ }^{2}$, Eliana Restrepo-Pineda ${ }^{3}$ and Alfonso Benítez-Páez ${ }^{1,5,6^{*}}$

\begin{abstract}
Background: Drug treatments and vaccine designs against the opportunistic human pathogen Pseudomonas aeruginosa have multiple issues, all associated with the diverse genetic traits present in this pathogen, ranging from multi-drug resistant genes to the molecular machinery for the biosynthesis of biofilms. Several candidate vaccines against $P$. aeruginosa have been developed, which target the outer membrane proteins; however, major issues arise when attempting to establish complete protection against this pathogen due to its presumably genotypic variation at the strain level. To shed light on this concern, we proposed this study to assess the $P$. aeruginosa pangenome and its molecular evolution across multiple strains.
\end{abstract}

Results: The $P$. aeruginosa pangenome was estimated to contain more than 16,000 non-redundant genes, and approximately $15 \%$ of these constituted the core genome. Functional analyses of the accessory genome indicated a wide presence of genetic elements directly associated with pathogenicity. An in-depth molecular evolution analysis revealed the full landscape of selection forces acting on the $P$. aeruginosa pangenome, in which purifying selection drives evolution in the genome of this human pathogen. We also detected distinctive positive selection in a wide variety of outer membrane proteins, with the data supporting the concept of substantial genetic variation in proteins probably recognized as antigens. Approaching the evolutionary information of genes under extremely positive selection, we designed a new Multi-Locus Sequencing Typing assay for an informative, rapid, and cost-effective genotyping of $P$. aeruginosa clinical isolates.

Conclusions: We report the unprecedented pangenome characterization of $P$. aeruginosa on a large scale, which included almost 200 bacterial genomes from one single species and a molecular evolutionary analysis at the pangenome scale. Evolutionary information presented here provides a clear explanation of the issues associated with the use of protein conjugates from pili, flagella, or secretion systems as antigens for vaccine design, which exhibit high genetic variation in terms of non-synonymous substitutions in $P$. aeruginosa strains.

Keywords: Molecular evolution, Pseudomonas aeruginosa, Pangenome, Non-synonymous substitutions, Synonymous substitutions, Genetic variation, Pathogenicity

\footnotetext{
*Correspondence: abenitez@cidbio.org

'Bioinformatics Analysis Group-GABi, Centro de Investigación y Desarrollo en

Biotecnología-CIDBIO, 111221 Bogotá DC, Colombia

${ }^{5}$ Centro de Investigación y Desarrollo en Biotecnología, Calle 64A \# 52-53

Int8 Of203, 111221 Bogotá DC, Colombia

Full list of author information is available at the end of the article
} 


\section{Background}

Humans are frequently infected by opportunistic pathogens that take advantage of their compromised immunological status to cause persistent and chronic infections. The Gram-negative bacterium Pseudomonas aeruginosa is one of those recurrent human pathogens. $P$. aeruginosa remains one of the most important pathogens in nosocomial infections, and it is often associated with skin, urinary tract, and respiratory tract infections [1]. Respiratory tract infections are of major relevance in cystic fibrosis patients, given that $P$. aeruginosa deeply affects their pulmonary function, causing life-threatening infections [2]. One of the better-known adaptive resistance mechanisms of $P$. aeruginosa to evade either the host immune response and drug therapy is its ability to form biofilms. The Pseudomonas aeruginosa biofilm is an extremely stable capsule-like structure constituted primarily of polysaccharides, proteins, and DNA, in which PsI exopolysaccharide seems to be a key player for biofilm matrix stability [3]. Quorum sensing signals promote the formation of $P$. aeruginosa biofilms, which minimizes the entry of antimicrobial compounds inside bacterial cells and hinders the recognition of pathogenassociated molecular patterns (PAMPs) by the host immune system [4]. Consequently, current treatments against $P$. aeruginosa fail to resolve infections before tissue deterioration occurs. To address this concern, more efficient alternatives to abolish $P$. aeruginosa infections have produced promising but not definitive results. Accordingly, several candidate $P$. aeruginosa vaccines have been developed by targeting outer membrane proteins (Opr), lipopolysaccharides (LPS), polysaccharides (PS), PS-protein conjugates, flagella, pili, and single or multivalent live-attenuated cells [5-9]. However, major issues in the development of a successful $P$. aeruginosa vaccine arise from the probable genotypic variation at the strain level, making $P$. aeruginosa a presumably antigenically variable organism. Results supporting this assumption have been reported, yielding genetic information from the $P$. aeruginosa genome. For example, genetic variability explored in multiple $P$. aeruginosa isolates from different regions of the world indicated that $\operatorname{pcr} V$, a member of the type III secretion system, exhibits limited genetic variation in terms of non-synonymous substitutions [10]. Although this type of analysis is informative, it provides only a very limited view of the genetic and evolutionary processes occurring at the genome level in $P$. aeruginosa and does not completely explain the failure to design and develop a successful vaccine against this human pathogen. Although antigen selection to design a $P$. aeruginosa vaccine is not a reported problem [11], to date, no genomic studies have correlated antigen genetic structure and variation with the effectiveness of antibody immunotherapy or vaccines, the efficacy of which remains elusive [11]. Moreover, enormous variation in the response against $P$. aeruginosa immunogenic proteins in patients with $P$. aeruginosa infections [12] could indicate that genetic factors from the pathogen and/or host could be responsible for the incomplete efficacy of candidate vaccines tested. In this fashion, this study aimed to i) better understand the genome structure and genetic variation exhibited by Pseudomonas aeruginosa, ii) link the genome variation information with past and future $P$. aeruginosa vaccine designs, and iii) present and validate new molecular markers for Multi-Locus Sequence Typing (MLST) based on the study of genes exhibiting a higher ratio of non-synonymous over synonymous substitution rate. To achieve these aims, a combined pangenome-wide and molecular evolution analysis was performed using up-to-date and genome-scale genetic information publicly available in the Pathosystems Resource Integration Center (PATRIC) database [13].

\section{Results and discussion}

Defining the Pseudomonas aeruginosa pangenome

A total of 181 genomes of $P$. aeruginosa strains were obtained through the public PATRIC database (see Methods and Additional file 1). The preliminary analysis of the $P$. aeruginosa genome size variability is shown in Table 1. The $P$. aeruginosa chromosome contains 6175 genes on average, with a distribution ranging from 5382 to 7170 genes per genome, indicating a variation of $13-16 \%$ in terms of gene content among all strains analysed. By using the genome-centred approximation to define the $P$. aeruginosa pangenome (see Methods), a total of 16,820 nonredundant genes were retrieved from those 181 genomes analysed. Almost one-third of the full set of genes constituting the $P$. aeruginosa pangenome, 5209 genes (31 \%), were found to be uniquely present, meaning that every strain approximately contributes 29 new genes to the Pseudomonas aeruginosa pangenome on average. Initially, these data fit well with a theoretical number of strainspecific new genes added to the pangenome when a new

Table 1 Main features of the Pseudomonas aeruginosa pangenome

\begin{tabular}{ll}
\hline Features analysed & P. aeruginosa pangenome \\
\hline Genomes & 181 \\
Total genes & $1,117,803$ \\
Average genome size & 6175 genes \\
Pangenome size (non-redundant genes) & 16,820 \\
Core genome & 2503 genes \\
Accessory genome & 9108 genes \\
Unique genes & 5209 \\
Average unique genes/strain & 16 \\
Gene families under positive selection & 233 \\
\hline
\end{tabular}


strain genome was sequenced, 33 for the Streptococcus agalactiae pangenome [14]. However, for a more precise calculation of genomic and functional features of the $P$. aeruginosa pangenome, we performed general methods described by Tettelin and co-workers to define bacterial pangenomes [15]. After an iterative and combinatorial process, our observed data was plotted as rarefaction curves following Heaps' law (Fig. 1a). Further information was extracted from the pangenome analysis regarding gene categorization. The core genome or extended core of genes was characterized as the set of genes present in all or almost all genomes analysed; in this manner, we established that the $P$. aeruginosa core genome contains approximately 2503 genes that are present in all 181 genomes studied, and they account for $15 \%$ of the pangenome. The graphical representation of the discovery rate for new genes at the core genome across the iterative analysis of $P$. aeruginosa strains for pangenome reconstruction is shown in Fig. 1b. We analyzed such data with power law $\left(n=\mathrm{kN}^{-\alpha}\right)$ finding and averaged alpha parameter of $2.36 \pm 0.49(\mathrm{CI}=2.27$ to 2.46$)$ indicating the $P$. aeruginosa pangenome is closed according to proposed postulates of Tettelin and co-workers [15]. Moreover, interpretation of these data with the exponential regression allowed to estimate and horizontal asymptote $(\theta) 10$ genes \pm 2.99 , indicating a small but finite number of new genes expected to be discovered with the study of new $P$. aeruginosa studies. A preliminary analysis regarding the distribution of some genes involved in lung infections like the biofilm-associated
A

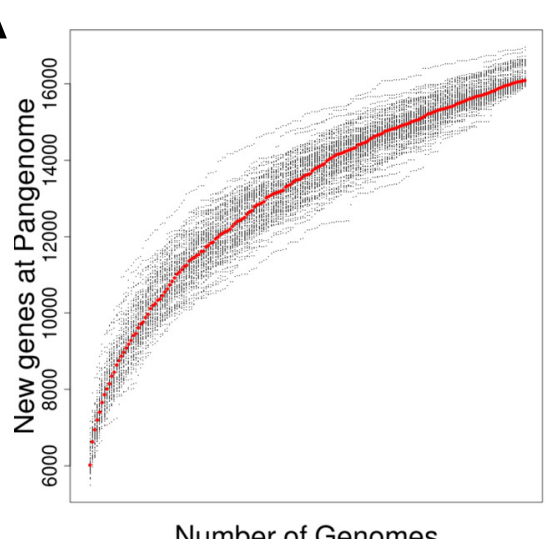

Number of Genomes
B

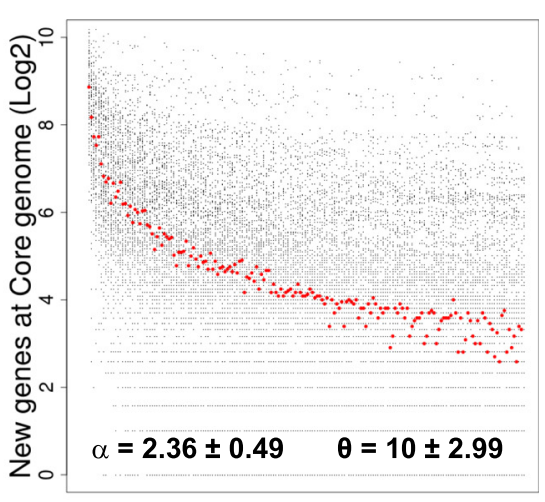

Number of Genomes

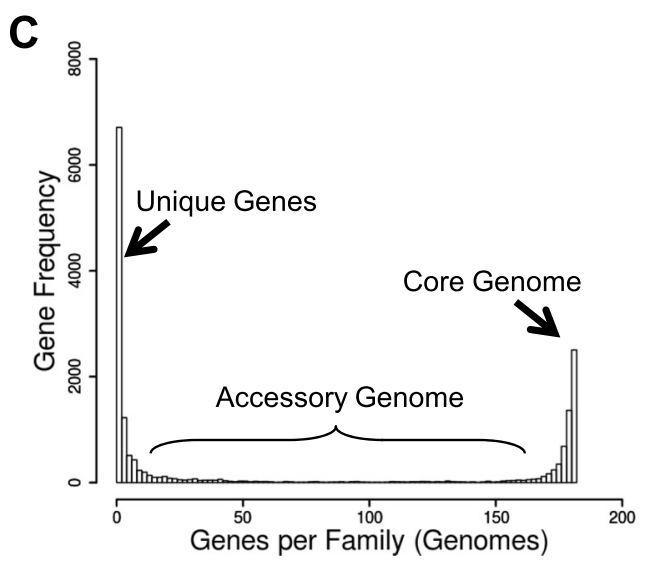

Fig. 1 The P. aeruginosa pangenome. a Rarefaction curve of the 200 different pangenomes calculated from random combinations of strains. Iterations and combinations are shown as the dots cloud indicating the total number of non-redundant genes included in the pangenome as genomes are included in the analysis. Red filled circles indicate the median of each iteration. $\mathbf{b}$ Decay function for new genes discovered during pangenome reconstruction. Iterations and combinations are shown as dots cloud indicating the number of new genes incorporated to core genome. Red filled circles indicate the median of each iteration. The power law alpha parameter shown inside the plot is the average of such values retrieved individually in each iteration after fitting $\pm s d$. The theta $(\theta)$ value was calculated from the horizontal asymptote where the exponential regression converges. c Histogram for the prevalence of different gene families of the pangenome. The 16,820 non-redundant gene families determined to be present in the $P$. aeruginosa pangenome were distributed according to their frequency across all strains analysed. Three gene categories are clearly distinguished, highlighting the core genome (gene families present in all strains analysed), the unique genes (genes present in only one strain), and the accessory genome (gene families exhibiting a variable frequency) 
(mifS, mifR, bamI, bdlA, bfiS, and $b f m R$ ) and antibiotic resistance genes (oprM, $a m p C$, $a m p D$, and PIB-1), and functionally annotated in the Pseudomonas Genome Database [16], has revealed that these genetic entities are present between 95 and $100 \%$ of the strains studied here. This indicates that such functions, and pathogenicity by extension, are encoded into the core genome of P. aeruginosa.

The set of genes, which were not included in the core genome or were unique (present in 1 genome), were referred to as the accessory genome; it included the $54 \%$ of genes found in the P. aeruginosa pangenome (Table 1). Interestingly, when we plotted the frequency of all pangenome genes present in different strains/genomes analysed (Fig. 1c), we found a similar distribution to that reported by Lapierre and Gogarten when they estimated the pangenome for more than 500 different bacterial genomes [17]. This distribution plot clearly demonstrated the characteristic distribution and frequency of different groups of the above-stated genes. In general terms, the $P$. aeruginosa pangenome exhibits a high level of genome variability, whereby only $40 \%(2503 / 6175)$ of its genome is constant, on average. Thus, the remaining $60 \%$ of $P$. aeruginosa genome is presented as a variable piece of DNA composed of a wide repertoire of genes and molecular functions. A very recent study has partially characterized the $P$. aeruginosa pangenome using a total of 20 different human- and environmental-derived strains. Their numbers in terms of average genome size and ORFs per strains are very close to those we show in the present study. However, they estimate the P. aeruginosa pangenome to have 13,527 with more than 4000 genes catalogued as the core genome [18]. The pangenome estimated in our study exceeds by more than 3000 genes to that reported by Hilker and co-workers as well as to that reported by Valot and co-workers [19]. This is totally expected given that the more genomes analyzed, the more probability to discover new genes, an assumption that is clearly exemplified in the Fig. 1a. Conversely, the core genome appear to be negatively affected by addition of new strains because the probability of sharing genes among strains decreases as new strains are incorporated to the study sample. This parameter intuitively is directly dependant of the number of strains used to calculate the core genome and their clonal relationship, which could strongly reduce gene diversity in the pangenome. Given that the multi-strain, iterative and combinatorial process used here to estimate the $P$. aeruginosa pangenome has produced a closed pangenome, we proposed that core genome for $P$. aeruginosa is composed of approximately 2500 genes. This number is notably lower than those proposed in very recent studies aiming the characterization of the $P$. aeruginosa pangenome as well [18-20]. However, none of those studies have produced a proper metrics indicating that their proposed pangenomes are closed. Therefore, our data represent the most accurate characterization of the $P$. aeruginosa pangenome supported in the analysis of more than 180 different strains throughout iterative and combinatorial approaches. Moreover, the metrics presented in here is very close to that early characterized for Escherichia coli, for which a core genome was defined to account 2200 genes [21].

Subsequently, we proceeded to perform a functional analysis with the full set of genes uniquely presented as well as other set of genes categorized by frequency in the $P$. aeruginosa pangenome. As a consequence, the nucleotide sequences of genes found to be present only in one $P$. aeruginosa strain were translated to amino acid sequences and then submitted to the Kyoto Encyclopedia of Genes and Genomes (KEGG) through the KEGG Automatic Annotation Server (KASS) for functional annotation at the protein level [22]. We retrieved only $14 \%$ (738 out of 5209 ) of the functional annotation for this set of genes, of which more than $59 \%$ (3075 out of 5209) comprises ORFs, encoding putative peptides shorter less than 100 aa in length. We explored the predominance of functions present in the 738 ORFs annotated at the KEEG Pathways level. Consequently, we found that in addition to proteins involved in more general functions, such as metabolic pathways (ko01100, 103 proteins) and the biosynthesis of secondary metabolites (ko01110, 30 proteins), proteins participating in more specific molecular tasks, such as the biosynthesis of antibiotics (ko01130, 22 proteins), the bacterial secretion system (ko03070, 20 proteins), ABC transporters (ko02010, 17 proteins), and two-component system (ko02020, 36 proteins), were frequently present as well. Among all of these proteins, we highlighted the presence of several members of the type II and IV secretion systems responsible for the secretion of bacterial toxins, proteins of the macrolide exporter system, and betalactamases and efflux pump proteins associated with betalactam resistance. Since such functional categories are found uniquely in different strains, this fact would support the idea that $P$. aeruginosa strains exhibit a wide variety of mechanisms to survive in several adverse environments being able to remain latently as reservoir of these genetic traits. Furthermore, this would have direct implication in emergence of multi-resistant and virulent strains since such genetic traits could all converge into single strains by horizontal transference mechanisms.

We further assessed the molecular functions of the portion of the $P$. aeruginosa accessory genome comprising genes between the 5th and 95th percentile of frequency $(9<$ accessory genome $<172)$ among all the genomes analysed. A total of 2605 proteins were submitted again to the KASS server, retrieving functional annotation for 735 (28 \%) of them. We found a similar predominance of the above-stated pathways, but we 
expanded our analysis to include the biosynthesis of amino acids (ko01230, 37 proteins) and amino sugar and nucleotide sugar metabolism (ko00520, 13 proteins). Strikingly, we found additional proteins involved in vancomycin resistance as well as proteins of the type I and VI secretion systems associated with the export of toxins, proteases, lipases and other effector proteins. A general view of the molecular functions confined to different categories of the $P$. aeruginosa pangenome is shown in Fig. 2. Comparison at the orthology level (Fig. 2) indicated that a high level of functional specificity exists in all gene categories of the $P$. aeruginosa pangenome, whereby $79 \%$ of annotated genes in the core genome are not present in other categories. This percentage remains high at $47 \%$ in unique genes and $49 \%$ in the accessory genome. Previous studies have shown similar results in terms of functional categories of core and accessory genomes partially defined for $P$. aeruginosa, where core genome is enriched in central metabolism functions and major cellular functions such as replication, transcription, and translation as well as other associated biosynthetic pathways $[19,20]$. At the KEGG functional module level, we disclosed some molecular pathways to be distinctive for every gene category in the pangenome. Table 2 summarizes those molecular pathways in which the $P$. aeruginosa core genome was found to contain a wide range of genes involved in either antibiotic biosynthesis and resistance. Therefore, functional characterization of the $P$. aeruginosa core genome would indicate that the infectivity and resistance are features intrinsically exhibited by any $P$. aeruginosa strain and that virulence and lethality would be confined to genetic traits encoded in the accessory genome.

\section{Molecular evolution in the Pseudomonas aeruginosa pangenome}

In addition to uncovering the genes and functions that confer distinctive features to $P$. aeruginosa strains, we explored the genetic variability in every gene family retrieved from its pangenome. This approach could provide evidence of how the $P$. aeruginosa genome evolves to evade the immune response as well as depict the level of variability thought to be the major cause of the lack of success in designing a effective vaccine. For more than 10,000 gene families containing at least 2 members, we calculated the synonymous (dS) and non-synonymous $(\mathrm{dN})$ rates, parameters indicative of the selection pressure on coding genes. The global distribution of $\mathrm{dS}$ and $\mathrm{dN}$ rates expressed as the omega value $(\omega=\mathrm{dN} / \mathrm{dS})$ across the $P$. aeruginosa pangenome is presented in Fig. 3a. Although the distribution of $\omega$ values fits well into a unimodal distribution, globally, it shows a shift-to-left distribution towards values lower than 1 with $\omega$ median $=0.1$. These data suggest that the $P$. aeruginosa coding genome is under purifying selection as a whole, in which synonymous substitutions are predominantly higher than nonsynonymous substitutions. The coding genes considered under positive selection must present $\omega>1(\mathrm{dN}>\mathrm{dS})$; however, at the initial stage, we performed more restrictive filtering, thus considering those genes that exhibited at least a 2-fold greater non-synonymous substitution rate than the synonymous substitutions $(\omega \geq 2)$. As a result, we retrieved a total of 230 genes (1.4\% of pangenome) for which 71 functional annotations (31\%) were recovered from the KASS server. We found a wide variability in terms of the molecular pathways for the genes under positive selection. Notably, among all genes under positive selection, we detected that some of them coded for proteins

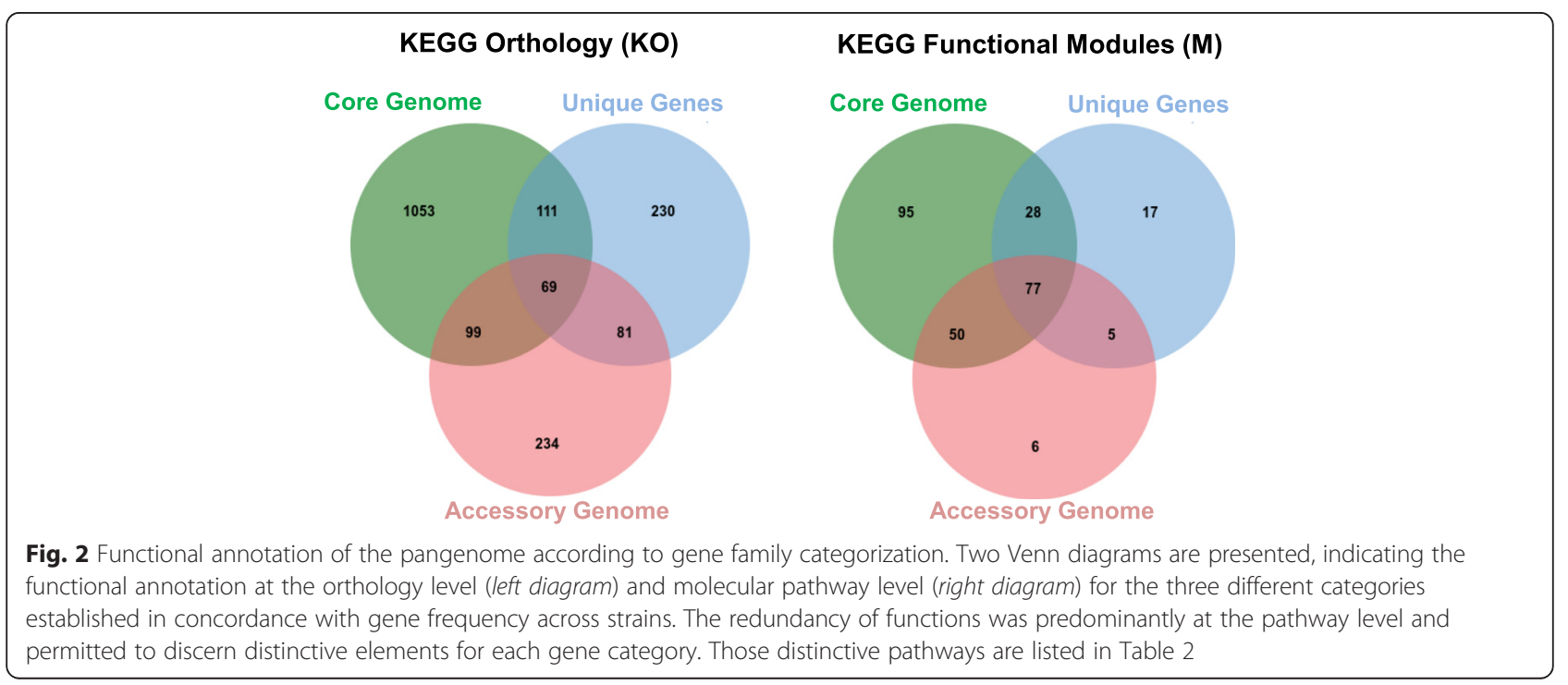


Table 2 KEGG functional modules distinctive for the $P$. aeruginosa pangenome gene category

\begin{tabular}{|c|c|c|c|c|}
\hline $\begin{array}{l}\text { KEGG module } \\
\text { number }\end{array}$ & Description & Genes & M00535 & Isoleucine biosynthesis \\
\hline \multicolumn{3}{|l|}{ Core genome } & M00113 & Jasmonic acid biosynthesis \\
\hline M00064 & $\begin{array}{l}\text { ADP-L-glycero-D-manno-heptose } \\
\text { biosynthesis }\end{array}$ & 2 & M00505 & $\begin{array}{l}\text { KinB-AlgB (alginate production) } \\
\text { two-component regulatory system }\end{array}$ \\
\hline \multirow[t]{2}{*}{ M00493 } & \multirow{2}{*}{$\begin{array}{l}\text { AlgZ-AlgR (alginate production) } \\
\text { two-component regulatory system }\end{array}$} & \multirow{2}{*}{1} & M00080 & Lipopolysaccharide biosynthesis \\
\hline & & & M00320 & Lipopolysaccharide export system \\
\hline M00235 & Arginine/ornithine transport system & 3 & M00255 & Lipoprotein-releasing system \\
\hline M00531 & Assimilatory nitrate reduction & 1 & M00116 & Menaquinone biosynthesis \\
\hline \multirow[t]{2}{*}{ M00475 } & \multirow{2}{*}{$\begin{array}{l}\text { BarA-UvrY (central carbon metabolism) } \\
\text { two-component regulatory system }\end{array}$} & \multirow[t]{2}{*}{2} & M00740 & Methylaspartate cycle \\
\hline & & & M00189 & Molybdate transport system \\
\hline M00086 & Beta-Oxidation & 1 & M00711 & Multidrug resistance, efflux pump MdtIJ \\
\hline $\begin{array}{l}\text { M00123, } \\
\text { M00573, M00577 }\end{array}$ & Biotin biosynthesis & 3 & M00115 & NAD biosynthesis \\
\hline M00364, M00366 & C10-C20 isoprenoid biosynthesis & 2 & M00144 & NADH:quinone oxidoreductase \\
\hline M00170, M00171 & C4-dicarboxylic acid cycle & 2 & M00471 & $\begin{array}{l}\text { NarX-NarL (nitrate respiration) } \\
\text { two-component regulatory system }\end{array}$ \\
\hline M00168 & CAM (Crassulacean acid metabolism) & 1 & M00622 & Nicotinate degradation \\
\hline $\begin{array}{l}\text { M00722, } \\
\text { M00727, M00728 }\end{array}$ & $\begin{array}{l}\text { Cationic antimicrobial peptide } \\
\text { (CAMP) resistance }\end{array}$ & 3 & M00615 & Nitrate assimilation \\
\hline M00256 & Cell division transport system & 1 & M00438 & Nitrate/nitrite transport system \\
\hline M00010 & Citrate cycle & 4 & M00439 & Oligopeptide transport system \\
\hline M00120 & Coenzyme A biosynthesis & 3 & M00209 & Osmoprotectant transport system \\
\hline M00338 & Cysteine biosynthesis & 1 & M00004, M00007 & Pentose phosphate pathway \\
\hline M00154, M00155 & Cytochrome c oxidase & 3 & M00024 & Phenylalanine biosynthesis \\
\hline M00417 & Cytochrome o ubiquinol oxidase & 3 & M00434 & $\begin{array}{l}\text { PhoR-PhoB (phosphate starvation } \\
\text { response) }\end{array}$ \\
\hline M00552 & $\begin{array}{l}\text { D-galactonate degradation, De } \\
\text { Ley-Doudoroff pathway }\end{array}$ & 2 & M00222 & Phosphate transport system \\
\hline M00596 & Dissimilatory sulfate reduction & 1 & M00501 & $\begin{array}{l}\text { PilS-PilR (type } 4 \text { fimbriae synthesis) } \\
\text { two-component regulatory system }\end{array}$ \\
\hline M00542 & EHEC/EPEC pathogenicity signature & 2 & M00133 & Polyamine biosynthesis \\
\hline M00008 & Entner-Doudoroff pathway & 1 & M00015 & Proline biosynthesis \\
\hline M00445 & $\begin{array}{l}\text { EnvZ-OmpR (osmotic stress response) } \\
\text { two-component regulatory system }\end{array}$ & 3 & M00247, M00258 & Putative $A B C$ transport system \\
\hline M00515 & $\begin{array}{l}\text { FlrB-FlrC (polar flagellar synthesis) } \\
\text { two-component regulatory system }\end{array}$ & 1 & M00193 & $\begin{array}{l}\text { Putative spermidine/putrescine } \\
\text { transport system }\end{array}$ \\
\hline M00729 & Fluoroquinolone resistance & 1 & M00046 & Pyrimidine degradation \\
\hline M00344, M00345 & Formaldehyde assimilation & 3 & M00053 & $\begin{array}{l}\text { Pyrimidine deoxyribonuleotide } \\
\text { biosynthesis, CDP/CTP }\end{array}$ \\
\hline M00497 & $\begin{array}{l}\text { GInL-GInG (nitrogen regulation) } \\
\text { two-component regulatory system }\end{array}$ & 1 & M00052 & $\begin{array}{l}\text { Pyrimidine ribonucleotide biosynthesis, } \\
\text { UMP }\end{array}$ \\
\hline M00605 & Glucose/mannose transport system & 2 & M00377 & Reductive acetyl-CoA pathway \\
\hline M00012 & Glyoxylate cycle & 1 & & (Wood-Ljungdahl pathway) \\
\hline M00050 & $\begin{array}{l}\text { Guanine ribonucleotide biosynthesis, } \\
\text { IMP }\end{array}$ & 3 & M00167 & Reductive pentose phosphate cycle \\
\hline M00259 & $\begin{array}{l}\text { MPP } \\
\text { Heme transport system }\end{array}$ & 1 & M00523 & $\begin{array}{l}\text { RegB-RegA (redox response) } \\
\text { two-component regulatory system }\end{array}$ \\
\hline M00045 & Histidine degradation & 4 & M00125 & Riboflavin biosynthesis, GTP \\
\hline M00226 & Histidine transport system & 1 & M00394 & RNA degradosome \\
\hline M00620 & Incomplete reductive citrate cycle & 3 & M00308 & $\begin{array}{l}\text { Semi-phosphorylative Entner-Doudoroff } \\
\text { pathway }\end{array}$ \\
\hline M00131 & Inositol phosphate metabolism & 1 & & Sulfate tra \\
\hline M00190 & Iron (III) transport system & 4 & M00436 & Sulfonate transport system \\
\hline
\end{tabular}

Table 2 KEGG functional modules distinctive for the $P$. aeruginosa pangenome gene category (Continued)
1 
Table 2 KEGG functional modules distinctive for the $P$. aeruginosa pangenome gene category (Continued)

\begin{tabular}{|c|c|c|}
\hline M00435 & Taurine transport system & 1 \\
\hline M00089 & Triacylglycerol biosynthesis & 2 \\
\hline M00332 & Type III secretion system & 2 \\
\hline M00025, M00040 & Tyrosine biosynthesis & 4 \\
\hline M00117, M00128 & Ubiquinone biosynthesis & 7 \\
\hline M00029 & Urea cycle & 3 \\
\hline M00651 & Vancomycin resistance & 2 \\
\hline M00241 & Vitamin B12 transport system & 1 \\
\hline M00660 & Xanthomonas spp. pathogenicity signature & 2 \\
\hline M00242 & Zinc transport system & 2 \\
\hline \multicolumn{3}{|l|}{ Accessory genome } \\
\hline M00502 & $\begin{array}{l}\text { Glrk-GlrR (amino sugar metabolism) } \\
\text { two-component regulatory system }\end{array}$ & 1 \\
\hline M00533 & Homoprotocatechuate degradation & 2 \\
\hline M00240 & Iron complex transport system & 3 \\
\hline M00005 & PRPP biosynthesis & 1 \\
\hline M00473 & $\begin{array}{l}\text { UhpB-UhpA (hexose phosphates uptake) } \\
\text { two-component regulatory system }\end{array}$ & 1 \\
\hline M00644 & Vanadium resistance & 1 \\
\hline \multicolumn{3}{|l|}{ Unique genes } \\
\hline M00653 & $\begin{array}{l}\text { AauS-AauR (acidic amino acids utilization) } \\
\text { two-component regulatory system }\end{array}$ & 1 \\
\hline M00500 & $\begin{array}{l}\text { AtoS-AtoC (cPHB biosynthesis) } \\
\text { two-component regulatory system }\end{array}$ & 1 \\
\hline M00450 & $\begin{array}{l}\text { BaeS-BaeR (envelope stress response) } \\
\text { two-component regulatory system }\end{array}$ & 1 \\
\hline M00104 & Bile acid biosynthesis & 1 \\
\hline M00581 & Biotin transport system & 1 \\
\hline M00569 & Catechol meta-cleavage & 4 \\
\hline M00582 & Energy-coupling factor transport system & 1 \\
\hline M00760 & Erythromycin resistance & 1 \\
\hline M00524 & $\begin{array}{l}\text { FixL-FixJ (nitrogen fixation) two- } \\
\text { component regulatory system }\end{array}$ & 1 \\
\hline M00713 & Fluoroquinolone resistance & 1 \\
\hline M00059 & Glycosaminoglycan biosynthesis & 1 \\
\hline M00499 & $\begin{array}{l}\text { HydH-HydG (metal tolerance) } \\
\text { two-component regulatory system }\end{array}$ & 1 \\
\hline M00714, M00645 & Multidrug resistance & 2 \\
\hline M00664 & Nodulation & 1 \\
\hline M00549 & Nucleotide sugar biosynthesis & 1 \\
\hline M00267 & $\begin{array}{l}\text { PTS system, N-acetylglucosamine-specific } \\
\text { Il component }\end{array}$ & 1 \\
\hline
\end{tabular}

Catalogue of the KEGG functional modules (M) distinctively found in three gene categories of the $P$. aeruginosa pangenome: core, accessory, and unique genes. The number of modules correlated with those numbers presented in Fig. 2 (Venn diagram on the right) with remarkable functions, such as VirB2 and VirB9 (K03197 and K03204, respectively). Both proteins are components of the type IV secretion system and are localized at the outer membrane. In the case of VirB2 proteins, the T-pilus protein controls attachment to different receptors on the host cell surface to deliver toxin effector molecules [23]. Attempts to distinguish the specific role of these proteins through homologue searching in the Uniprot database have retrieved unclear results given that amino acid sequences of VirB2 and VirB9 from P. aeruginosa pangenome matched primarily with conjugal transfer proteins from A. tumefaciens (identity $\sim 40$ over $70 \%$ of the protein length), but also with toxin liberation protein F from $B$. pertussis (identity $\sim 28$ over $80 \%$ of the protein length). In any event, the VirB2 and VirB9 proteins must be exposed on the cell surface of pathogens making possible the $P$. aeruginosa be recognized by the host immune system and triggering a specific response against these potential antigens, thus promoting immune memory against this pathogen. The antigenicity of VirB2 and VirB9 proteins is further supported by their high rate of nonsynonymous substitutions observed across different strains analysed, which would be result of the strong selection forces from the host immune system. Notwithstanding, we cannot discard these high rates of nonsynonymous substitutions appear as response of phage predation. In this last scenario, the information retrieved in the present study regarding the set of genes under strong positive selection can be also useful to design bacteriophage-based therapies which have already been tested in $P$. aeruginosa [24]. Similarly, other outer membrane-bound proteins, such as the flippase MurJ (K03980) and the flagellin FlgF (K02391), which have been associated with virulence and pathogenicity $[25,26]$, exhibited a higher rate of non-synonymous substitutions than synonymous substitutions .

Strong selection forces from the immune response or environmental pressure were also detected in a set of $P$. aeruginosa genes tightly linked with virulence in other human pathogens. Therefore, we observed positive selection in the following genes: the PsrtC (K08303) homologue, a protease involved in mucus degradation during $H$. pylori infection (pathway ko05120); the MprF and ParR homologues (KO14205 and K18073, respectively), proteins involved in the cationic antimicrobial peptide (CAMP) resistance in Gram-positive and Gram-negative bacteria (ko1503), respectively; the PstS homologue (K02040), an integral membrane ABC phosphate transporter that modulates the TLR4 response during $M$. tuberculosis infection (ko5152); the T. brucei ICP homologue (14475), a protein involved in immunosupression by modulating the degradation of IgGs (ko5143); and the RNA polymerase sigma-54 factor (K03092), which is associated with the $V$. cholera 
A

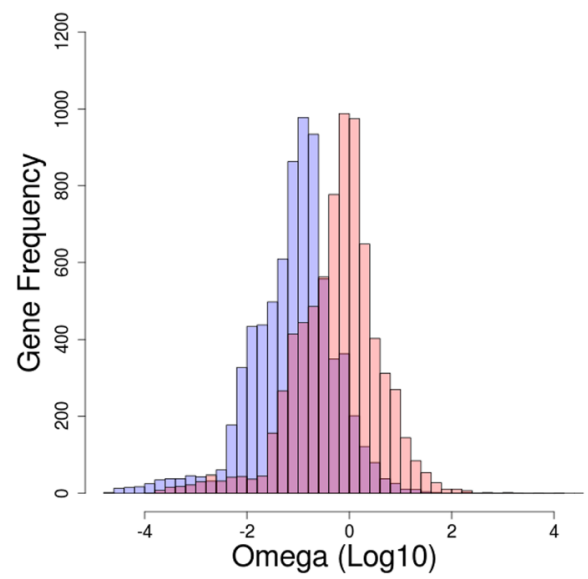

B

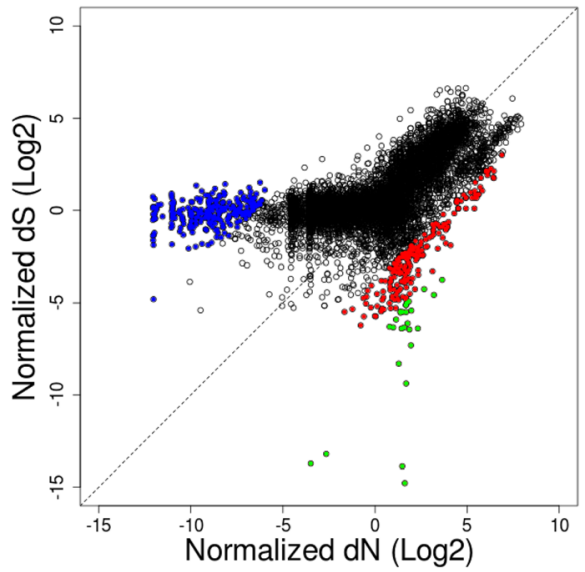

Fig. 3 Molecular evolution of the $P$. aeruginosa pangenome. a Histogram showing the distribution of omega ( $\omega$ ) values across the $P$. aeruginosa pangenome. The light blue histogram shows the original distribution with the tendency towards values indicating purifying selection (shift to left from neutrality). The superposed light red histogram indicates the Z-scores for the selection of genes with $\omega$ significantly different than 1 . Those with significant $\omega<1$ were considered to be under strong purifying selection for functional analysis, and those with significant $\omega>1$ were selected to be under strong positive selection for the MLST approach. $\mathbf{b}$ Scatter plot to represent the distribution of normalized dN and dS rates for all gene families detected in the $P$. aeruginosa pangenome. Gene families under strong purifying selection are highlighted in blue, whereas gene families under positive selection $(\omega>2)$ are highlighted in red. The set of gene family candidates for MLST under strong positive selection are highlighted in green. The diagonal dashed line indicates the boundary for neutrality

pathogenic cycle to control the expression of motor components of flagella (ko5111).

Given the low level of functional annotation for genes under positive selection, we performed an additional quantitative assessment to determine protein domain enrichment in the group of proteins under positive selection using the Simple Modular Architecture Research Tool (SMART) and the Protein Family database (Pfam) nomenclature systems. Once the inventory of SMART and Pfam domains contained in the entire $P$. aeruginosa pangenome was assessed, we performed a Fisher's exact test for $2 \times 2$ contingency tables to verify the significant over-representation of Pfam/SMART domains in the proteins under positive selection with respect to the pangenome. We observed the presence and prevalence of 4090 different protein domains from both the SMART and Pfam classification in the P. aeruginosa pangenome. Forty-four of these 4090 domains were found to be over-represented in the proteins exhibiting positive selection (Table 3). Among them, we observed a high frequency of membrane-bound proteins acting as transporters or receptors. Some of the functions overrepresented in Table 3 agree with some stated from previous analyses in which membrane proteins (transporters and/or receptors) as well as the Sigma-54 factor seem to be under positive selection in $P$. aeruginosa. Interestingly, we observed the presence of proteins related with either 16S RNA and ribosomal protein methylation
(Table 3). We detected such patterns of molecular evolution in this class of proteins previously, but in different human pathogens [27]. Although we cannot shed light on the meaning of this type of evolution in these proteins given their function, we hypothesized that they might influence the translation process to modulate the expression of a certain set of proteins directly or indirectly involved in pathogenesis. Recent studies on rRNA methylation indicate that they play a meaningful role in decoding function [28-30]. Indeed, some of them have been directly involved with virulence [31].

When we attempted a similar analysis in a counterpart set of proteins under purifying or negative selection $(\omega<1)$, the biased distribution of omega values across the $P$. aeruginosa pangenome (Fig. 3a) made it difficult to set up a suitable threshold to recover proteins under this type of selection. Therefore, we obtained Z-scores of both the $\mathrm{dN}$ and $\mathrm{dS}$ rates (Fig. 3a, light red histogram), thus reaching a normal distribution around $\omega=1$ (neutrality). Using this normalized distribution of $\omega$ values, we could determine those genes with evolution significantly different $(p \leq 0.05)$ from neutrality $(\omega=1)$ towards a strong negative selection (lowest $\omega$ values). As a result, we found a group of 268 proteins/genes under negative selection, the $\mathrm{dN}$ and $\mathrm{dS}$ rates of which are plotted in Fig. $3 \mathrm{~b}$ (see the blue points distribution). The quantitative assessment to determine protein domain enrichment indicated that more than 130 SMART and/or Pfam domains were over- 
Table 3 Domain enrichment in proteins under positive selection

\begin{tabular}{|c|c|c|}
\hline SMART/Pfam domain & Description & Fisher's test \\
\hline Chromate_transp & Probably act as chromate transporters in bacteria & 0.0000 \\
\hline Sulfatase & Present in esterases hydrolysing steroids, carbohydrates and proteins & 0.0020 \\
\hline PepSY_TM & Conserved transmembrane helix found in bacterial protein families & 0.0041 \\
\hline $\operatorname{PrmA}$ & Present in the Ribosomal protein $L 11$ methyltransferase & 0.0123 \\
\hline Cons_hypoth95 & Present in 165 RNA methyltransferase D & 0.0166 \\
\hline MTS & Present in the $16 \mathrm{~S}$ RNA methyltransferase $\mathrm{C}$ & 0.0182 \\
\hline DUF1329 & Putative outer membrane lipoprotein & 0.0215 \\
\hline DUF4102 & Putative phage integrase & 0.0235 \\
\hline CHASE & Extracellular domain of bacterial transmembrane receptors & 0.0284 \\
\hline G3P_acyltransf & Enzymes converting glycerol-3-phosphate into lysophosphatidic acid & 0.0284 \\
\hline AceK & Bacterial isocitrate dehydrogenase kinase/phosphatase protein & 0.0284 \\
\hline Choline_sulf_C & C-terminus of enzyme producing choline from choline-O-sulfate & 0.0284 \\
\hline DUF2165 & Unknown function & 0.0284 \\
\hline DUF2909 & Unknown function & 0.0284 \\
\hline DUF3079 & Unknown function & 0.0284 \\
\hline DUF444 & Unknown function & 0.0284 \\
\hline DUF533 & Unknown function; integral membrane protein & 0.0284 \\
\hline DUF791 & Unknown function & 0.0284 \\
\hline DUF972 & Unknown function & 0.0284 \\
\hline Glu_cys_ligase & Enzyme carrying out the first step of glutathione biosynthesis & 0.0284 \\
\hline Herpes_UL6 & Present in proteins similar to herpes simplex UL6 virion protein & 0.0284 \\
\hline His_kinase & Membrane sensor, a two-component regulatory system & 0.0284 \\
\hline Inhibitor_142 & Protease inhibitor & 0.0284 \\
\hline PPDK_N & Present in enzymes catalysing the conversion of pyrophosphate to PEP & 0.0284 \\
\hline Sigma54_AID & Activating interacting domain of the Sigma-54 factor & 0.0284 \\
\hline Sigma54_CBD & Core binding domains of the Sigma-54 factor & 0.0284 \\
\hline Sigma54_DBD & DNA binding domain of the Sigma-54 factor & 0.0284 \\
\hline PAS, PAS 4/9 & Present in signalling proteins working as signal sensors & 0.0330 \\
\hline MFS & Major Facilitator Superfamily of small molecule transporters & 0.0359 \\
\hline Autoind_synth & Autoinducer synthase involved in quorum-sensing response & 0.0423 \\
\hline$A z \mid C$ & Putative protein involved in branched-chain amino acid transport & 0.0423 \\
\hline Chitin_bind & Present in carbohydrate-active enzymes (glycoside hydrolases) & 0.0423 \\
\hline DUF3299 & Unknown function & 0.0423 \\
\hline PTS_EIIC / IIB & Phosphoenolpyruvate-dependent phosphotransferase system & 0.0423 \\
\hline TctC & Member of the tripartite tricarboxylate receptors & 0.0423 \\
\hline UPF0004 & Domain found in tRNA methythiotransferases & 0.0423 \\
\hline
\end{tabular}

The SMART and Pfam domains are presented in a non-redundant manner. Function description was recovered from annotations in SMART or Pfam databases. Fisher's test values correspond to $p$-values $(p \leq 0.05)$, supporting the over-representation of the corresponding domain in the set of proteins under positive selection

represented in this set of proteins, and as expected, most of them were related to the central functions of cell maintenance, such as translation (ribosome proteins, tRNA biogenesis, amino acid starvation response), carbohydrate metabolism, amino acid biosynthesis and transport, and respiration.

\section{New high variability markers for multi-locus sequence typing of $P$. aeruginosa strains}

Characterization of the $P$. aeruginosa pangenome offers not only critical information about the molecular functions and prevalence of certain genes across multiple strains analysed but also information about the level of 
genetic variability at the strain level. A molecular evolution approach retrieved a large set of genes/proteins under positive selection in $P$. aeruginosa. At the same time, such genes could be used for genotyping aims to associate certain genetic variants with pathogenicity and virulence traits. As a consequence, we selected and tested some $P$. aeruginosa genes in a MLST strategy to discern phylogenetic relationships among a large number of PATRIC reference strains analysed and six $P$. aeruginosa aminoglycoside and carbapenem-resistant strains isolated from patients who acquired healthcare-associated infections in a clinic located outside the metropolitan area of Medellin, Antioquia, Colombia.

We narrowed down the list of MLST candidates by selecting the genes that had the following characteristics: i) present in at least $95 \%$ of the strains explored at the sequence level (frequency $\geq 172$ ); ii) exhibiting omega values significantly higher than 1 (Fig. $3 \mathrm{~b}, p \leq 0.05, \omega>15$ ); and iii) short enough to facilitate Sanger sequencing in a few reactions. Of the 27 genes/proteins showing significant positive selection, we finally selected four genes, the features of which are depicted in Table 4. After amplification and Sanger sequencing of selected genes in our six $P$. aeruginosa isolates, we combined that genetic information with that completely available for $170 \mathrm{P}$. aeruginosa strains, thus building a multiple sequence alignment almost $3000 \mathrm{bp}$ in length for a total of 176 different strains. Using maximum likelihood approaches, we reconstructed the phylogenetic relationships among all strains and retrieved the phylogenetic tree showed in Fig. 4. Our six local isolates were positioned in three different clades, where isolate 49 was closely related to the highly virulent $P$. aeruginosa PA14 strain, representing the most common clonal group worldwide [32]. By contrast, isolate 77 was related to several strains, including the multi-drugresistant $P$. aeruginosa NCGM2.S1 [33] and the cytotoxic corneal isolate $P$. aeruginosa 6077 [34]. Finally, the 30-1, 42-1, 45, and 04 isolates presented a close relationship and were related to the multi-drug resistant $P$. aeruginosa VRFPA02 isolate from India [35].

Based on the best evolutionary model fitted to the nucleotide substitution pattern observed for these markers $(\operatorname{TrN}+\mathrm{I}+\mathrm{G})$, a proportion of invariable sites of 0.9080 was obtained, thus indicating that more than 250 polymorphic sites are present in our MLST approach. Moreover, gamma distribution parameters $(0.5060)$ is indicative of few hot-spots with high substitution rates [36]. In this fashion, we provided support to use the highly variable genetic markers reported here for MLST to produce an initial, fast, and cost-effective genotyping for $P$. aeruginosa strains of clinical interest. To compare if the evolutionary history of $P$. aeruginosa strains is equally represented by of our proposed MLST markers in comparison with that inferred by using common MLST markers [37, 38], we reconstructed a phylogeny using similar approaches and DNA sequences corresponding to seven housekeeping genes: acs $A$, aroE, guaA, mutL, nuoD, ppsA, and trpE. The resulting tree showed not deep topology differences when compared to that created from our proposed MLST approach (data not shown). This indicate that the new molecular markers proposed in this study for genotyping aims could be used to infer the evolutionary history of $P$. aeruginosa strains.

\section{Conclusions}

High-throughput sequencing technology has permitted the analysis of the genetic identity of a vast number of microorganisms, an applied science especially relevant to studying human pathogens and their virulence and pathogenicity traits in depth. Here, we have performed a reverse vaccinology approach using a large amount of genetic information available in the PATRIC database to determine the genetic elements of Pseudomonas aeruginosa to be probably targeted in future clinical studies aiming new vaccine designs. We have extensively described the $P$. aeruginosa pangenome in terms of the effective number of non-redundant genes present in this bacterial species by analysing more than 180 different strain genomes. We outlined the genomic variability of this human pathogen, demonstrating that approximately $60 \%$ of the $P$. aeruginosa genome is variable across strains, with the remaining genome encoding genes that are involved in central functions, such as virulence, resistance, toxicity and pathogenicity.

We have identified major genetic pieces of the core and accessory genome in $P$. aeruginosa. Approximately $15 \%(2503 / 16,820$ genes $)$ of the pangenome was found

Table 4 Potential genetic markers for MLST in P. aeruginosa strains

\begin{tabular}{|c|c|c|c|c|}
\hline Gene family $^{a}$ & Function $^{\mathrm{b}}$ & Omega $(\omega)$ & Length (bp) & Strain frequency ${ }^{c}$ \\
\hline 3333 & Chitin binding protein & 108 & 1170 & $98.9 \%(179)$ \\
\hline 3675 & Flagellar basal-body rod protein FlgF & 5884 & 750 & $99.5 \%(180)$ \\
\hline 4766 & Predicted branched-chain amino acid permease AzIC & 86 & 763 & $96.7 \%(175)$ \\
\hline 5348 & Unknown function & 32 & 573 & $99.5 \%(180)$ \\
\hline
\end{tabular}

${ }^{a}$ Nomenclature according to pangenome gene inventory

${ }^{b}$ Function inferred from KEGG, SMART, and/or BLAST-based search

${ }^{c}$ Number of strains carrying respective genes are denoted in parenthesis 


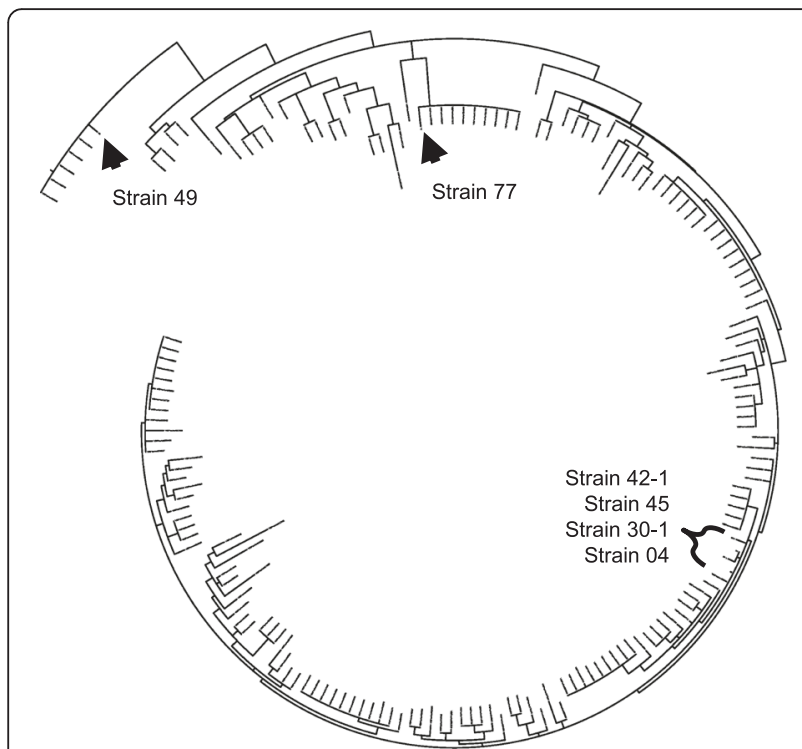

Fig. 4 Circular phylogenetic tree showing the genetic relationships among 170 reference PATRIC strains and our six $P$. aeruginosa isolates. The phylogenetic tree was built from the best evolutionary model explaining evolution at the concatenated gene families 3333, 3675,4766 , and 5348 after a sequential likelihood ratio test [54]. A total of 176 P. aeruginosa strains are located in the tree, and the localization of our clinical isolate is indicated. A close view of this tree permitted us to infer relationships among our clinical isolates with virulent and multi-drug resistant strains

to constitute the core genome and was present in $100 \%$ of the strains studied, accomplishing general molecular functions for cell maintenance such as replication, translation, transcription, central metabolism, electron transport chain, amino acid biosynthesis and transport, nucleotide biosynthesis, cell wall synthesis and maintenance, and cell division. Conversely, the accessory genome exhibited a comprehensive variety of functions, ranging from a wide spectrum of antibiotic resistances to a specialized secretion system delivering toxins and effector proteins potentially harmful for host cells. However, pathogenicity traits were also observed in the distinctive KEGG pathways revealed for the core genome.

Although this is not the first report to describe the pangenome for a single bacterial species [14, 21, 39, 40], and other very recent studies have attempted to determine the $P$. aeruginosa pangenome [18-20], this report is the first to describe a closed $P$. aeruginosa pangenome at very large scale, including almost 200 bacterial genomes from this human pathogen and performing a pangenome-scale molecular evolutionary analysis. Our study fits well with previous and general genomic characterizations of this human pathogen [18-20,41], and it definitely expands our knowledge about the evolutionary mechanisms of $P$. aeruginosa pathogenesis. This study aimed to reveal the evolutionary processes occurring at the pangenome level in $P$. aeruginosa that could explain the failure to design and develop of a successful vaccine against this human pathogen as well as provide an understanding of the molecular mechanisms that drive the evasion of the host immune system. We observed that the $P$. aeruginosa genome is globally under purifying selection, given the distribution of omega values $(\omega=\mathrm{dN} /$ $\mathrm{dS}$, median $\sim 0.1$ ) discerned for every gene family present in its pangenome. This result was further supported by the finding that there are 10-fold more genes under strong purifying selection than strong positive selection (significantly different to neutrality, $p \leq 0.05$ ). Although we found that the $P$. aeruginosa pangenome evolves to purifying selection as a whole, we distinguished some genes and functions predominantly present in the reduced set of genes under positive selection. As a consequence, a considerable number of proteins located at the outer membrane, such as those associated with receptor and transporter functions, were identified to have an increased rate of non-synonymous substitutions. These data corroborated our results based on KEGG functional analysis, which described an ample group of surfaceexposed proteins under strong selection forces from the immune response or environmental pressure.

For the first time, pangenome-scale evolutionary information is presented to support the design of new $P$. aeruginosa vaccines. In this fashion, failures when using protein conjugates from pili, flagella, or secretions systems $[5,7,9,11]$ are partially explained by the data presented here, which indicates the presence of a high genetic variation in this class of proteins in terms of non-synonymous substitutions, a fact that has been described previously but at very lower scale [42, 43].

Finally, we further explored the genetic information derived from our molecular evolution analyses and proposed a set of four new polymorphic genetic markers for MLST. We demonstrated that these markers contain an adequate proportion of hotspots for variation, exhibiting high nucleotide substitution rates. Using these four loci, we discerned the genetic identity of 6 local isolates of $P$. aeruginosa and related them with the resistance and virulence traits carried in reference strains.

\section{Methods}

\section{Pangenome-wide analysis}

Genome information from $P$. aeruginosa strains was downloaded via the $\mathrm{ftp}$ server from the PATRIC database [13]. A set of 181 available genomes (ffn files) was retrieved from the PATRIC database, April 2014 release. Estimation of the Pseudomonas aeruginosa pangenome size was assessed in a similar manner to that previously reported as genome- and gene-oriented methods using iterative and combinatorial approaches [14, 15, 17]. Briefly, a BLAST-based iterative method was used to extract the full set of non-redundant genes representing 
the $P$. aeruginosa pangenome. A single iteration consisted in a random selection of a strain as pangenome primer, then the remaining set of strain were randomly incorporated to the pangenome. The above process was calculated over 200 iterations with random permutation of the strain order in every iterative step. A rarefaction curve was plotted with all data generated and consisted in 200 different measures throughout a sequential addition of 181 different strains. Pangenome metrics was also obtained in iterative manner with data fitting to the power law as previously stated [15]. Power regression was calculated individually for each iteration of pangenome reconstruction $(n=200)$ and plotted in R v.3.1.2 with the "igraph" package (https://cran.r-project.org/). Alpha parameter from the $n=\mathrm{kN}^{-\alpha}$ power regression, indicating whether pangenome is open or closed, was calculated individually with least squares fit of the power law to the number of new genes discovered at core genome according to tettelin and coworkers [15]. Therefore, the global alpha value for the $P$. aeruginosa pangenome was determined as the mean of all 200 different alpha values generated $\pm \mathrm{sd}$ with the confidence interval at 0.95 level. Finally, the set of non-redundant genes obtained was used to explore their occurrence pattern in the 181 P. aeruginosa genomes through BLASTN-based comparisons [44, 45].

\section{Molecular evolution analysis}

The full set of ORFs constituting the $P$. aeruginosa pangenome was used to search homologues in all genomes analysed, and multiple sequence alignments were built using refined and iterative methods [46, 47]. The synonymous and non-synonymous substitution rates were calculated in a pairwise manner using approximate methods [48] and by correcting for multiple substitutions [49]. Omega values $(\omega)$ were computed as the averaged ratio of $\mathrm{dN} / \mathrm{dS}$ rates from multiple comparisons, and genes under strong positive selection were selected when $\omega \geq 2$. The Z-score of $\omega$ values was computed to depict functions of genes under strong purifying selection and potential MLST genetic markers under strong positive selection $(p \leq 0.05)$. Large-scale analyses of pairwise comparisons, statistical analysis, and graphics were performed using R v3.1.2 (https://cran.r-project.org/).

\section{Functional genomics analysis}

Functional annotation of genes was performed using the KEGG Automatic Annotation Server for KEGG Orthology [22]. KEGG functional modules and ontologies were explored in the KEGG BRITE database [50]. Functional domains present in genes of interest were assigned using Perl scripting for batch annotation (http://smart.emblheidelberg.de/help/SMART_batch.pl) against the Simple Modular Architecture Research Tool (SMART) together with Pfam classification [51, 52]. Fisher's exact test with a false discovery rate (FDR) for $2 \times 2$ contingency tables to measure enrichment of Pfam/SMART domains was performed using R v3.1.2 (https://cran.r-project.org/). Venn diagrams were drawn using the jvenn server [53].

\section{Multi-locus sequence typing}

The six $P$. aeruginosa strains (labelled as 04, 30-1, 42-1, 45,49 , and 77) were isolated from patients who acquired healthcare-associated infections at a clinic located outside the metropolitan area of Medellin, Antioquia, Colombia. This study was approved by the ethics committee of the Fundación Clínica del Norte Hospital (Bello, Antioquia, Colombia). The six isolates, previously characterized for multi-drug resistance, were kindly donated to the scientists of the Bacteria \& Cancer Researching Group of the Faculty of Medicine, University of Antioquia, Colombia. The genomic DNA from $P$. aeruginosa multi-drug resistant strains was extracted using a GeneJET Genomic DNA Purification Kit (Thermo Scientific, Waltham, MA, USA). The reference sequences of $P$. aeruginosa PA01 for the four markers selected to perform MLST were downloaded from a public database [GenBank: AE004091.2: region 930623 to 931822 (family 3333), region 1167488 to 1168237 (family 3675), region 2230183 to 2229425 (family 4766), region 2935851 to 2936423 (family 5348)]. Primers were designed to amplify the complete sequence of each gene, and the Polymerase Chain Reaction (PCR) proceeded with 28 cycles of amplification using Phusion ${ }^{\circ}$ High-Fidelity DNA Polymerase (Thermo Scientific, Waltham, MA, USA) and $50 \mathrm{ng}$ of genomic DNA. PCR products were isolated using a GeneJet PCR Purification Kit (Life technologies, Carlsbad, CA, USA), and both strands were sequenced by the Sanger automatic method in an ABI $3730 \mathrm{xl}$ instrument (Stab Vida Inc., Caparica, Portugal). Base calling and genetic variants were manually explored using the delivered $a b 1$ files and FinchTV viewer (Geospiza Inc. Perkin Elmer, Waltham, MA, USA). Assembled sequences from both strands were obtained and concatenated to respective reference sequences obtained from the PATRIC genomes analysed. Sequences belonging to the respective gene family were aligned using iterative methods $[46,47]$, and alignments were concatenated to perform phylogenetic analysis. The sequential likelihood ratio test was carried out to detect the evolutionary model that better explained genetic variation in all genes integrated in the MLST approach. For that reason, we used the jModelTest tool [54], and model selection was completed by calculating the corrected Akaike Information Criterion (cAIC). The MLST tree was constructed using the Interactive Tree Of Life (iTOL) tool $[51,55]$ and the phylogeny obtained using the TrN $+\mathrm{I}+\mathrm{G}$ model. For comparisons aims, we compiled genetic information from seven MLST markers commonly 
used in $P$. aeruginosa genotyping being the housekeeping genes: acs $A$, aroE, guaA, mutL, nuoD, ppsA, and trpE $[37,38]$. Aligned sequences were concatenated and phylogenetically analyzed with the jModelTest tool as well. Tree topology generated from this conventional MLST markers was compared with that obtained using the new MLST markers proposed in this study.

\section{Availability of supporting data}

The features of the Pseudomonas aeruginosa strains used in this study are included in the Additional file 1. All the DNA sequences derived from PCR amplification and Sanger sequencing of the four MLST studied here for the $P$. aeruginosa clinical isolates were submitted to the GenBank through BankIt server [GenBank: KU214214 to KU214237].

\section{Additional file}

Additional file 1: Metadata associated with the 181 strains used in this study to reconstruct the P. aeruginosa pangenome. All the information regarding the genome size, ORF predicted, RNA genes, host, geographic location, virulence, and resistance genes were recovered from PATRIC database. NA indicates no information available. (PDF 808 kb)

\section{Competing interests}

The authors declare that they have no competing interests.

\section{Authors' contributions}

ABP designed and directed this study. JMR and ABP performed the pangenome, molecular evolution, and phylogenetic analyses. ERP and AMR obtained the $P$. aeruginosa clinical isolates. JMR, AMR, and MC performed $P C R$ techniques. JMR and SCB curated the sequences from Sanger automatic sequencing. JMR, SCB, and ABP prepared the manuscript. All authors read and approved the final version of this manuscript.

\section{Acknowledgements}

The authors give thanks to the Colombian Agency for Science, Technology, and Innovation (Colciencias) and the National Fund for Science, Technology, and Innovation "Francisco José de Caldas" for grant 5817-5693-4856 to ABP and grant 1115-5693-3375 to ERP. The authors also thank the "Clinica Antioquia" microbiology laboratory staff, who donated the clinical isolates for the MLST studies. The JMR M.Sc. fellowship was supported by the Colombian Agency for Science, Technology, and Innovation (Colciencias) with funds of the 5817-5693-4856 grant.

\section{Author details}

${ }^{1}$ Bioinformatics Analysis Group-GABi, Centro de Investigación y Desarrollo en Biotecnología-CIDBIO, 111221 Bogotá DC, Colombia. ${ }^{2}$ Grupo GEBIOMIC, FCEN, Universidad de Antioquia, Medellín, Colombia. ${ }^{3}$ Grupo Bacterias y Cáncer, Universidad de Antioquia, Medellín, Colombia. ${ }^{4}$ Grupo Biociencias, Institución Universitaria Colegio Mayor de Antioquia, Medellín, Colombia. ${ }^{5}$ Centro de Investigación y Desarrollo en Biotecnología, Calle 64A \# 52-53 Int8 Of203, 111221 Bogotá DC, Colombia. ${ }^{6}$ Microbial Ecology, Nutrition \& Health Research Unit, Agrochemistry and Food Technology Institute (IATA-CSIC), 46980 Paterna-Valencia, Spain.

Received: 22 May 2015 Accepted: 5 January 2016

Published online: 12 January 2016

\section{References}

1. Lavoie EG, Wangdi T, Kazmierczak BI. Innate immune responses to Pseudomonas aeruginosa infection. Microbes Infect. 2011;13(14-15):1133-45.
2. Hauser $A R$, Jain M, Bar-Meir M, McColley SA. Clinical significance of microbial infection and adaptation in cystic fibrosis. Clin Microbiol Rev. 2011;24(1):29-70.

3. Ma L, Conover M, Lu H, Parsek MR, Bayles K, Wozniak DJ. Assembly and development of the Pseudomonas aeruginosa biofilm matrix. PLoS Pathog. 2009;5(3), e1000354.

4. Alhede M, Bjarnsholt T, Givskov M, Alhede M. Pseudomonas aeruginosa biofilms: mechanisms of immune evasion. Adv Appl Microbiol. 2014;86:1-40.

5. Doring G, Meisner C, Stern M. A double-blind randomized placebocontrolled phase III study of a Pseudomonas aeruginosa flagella vaccine in cystic fibrosis patients. Proc Natl Acad Sci U S A. 2007;104(26):11020-5.

6. Lang AB, Rudeberg A, Schoni MH, Que JU, Furer E, Schaad UB. Vaccination of cystic fibrosis patients against Pseudomonas aeruginosa reduces the proportion of patients infected and delays time to infection. Pediatr Infect Dis J. 2004:23(6):504-10.

7. Horn MP, Zuercher AW, Imboden MA, Rudolf MP, Lazar $\mathrm{H}$, Wu H, et al. Preclinical in vitro and in vivo characterization of the fully human monoclonal IgM antibody KBPA101 specific for Pseudomonas aeruginosa serotype IATS-011. Antimicrob Agents Chemother. 2010;54(6):2338-44.

8. Kamei A, Coutinho-Sledge YS, Goldberg JB, Priebe GP, Pier GB. Mucosal vaccination with a multivalent, live-attenuated vaccine induces multifactorial immunity against Pseudomonas aeruginosa acute lung infection. Infect Immun. 2011;79(3):1289-99.

9. Campodonico VL, Llosa NJ, Bentancor LV, Maira-Litran T, Pier GB. Efficacy of a conjugate vaccine containing polymannuronic acid and flagellin against experimental Pseudomonas aeruginosa lung infection in mice. Infect Immun. 2011;79(8):3455-64.

10. Lynch SV, Flanagan JL, Sawa T, Fang A, Baek MS, Rubio-Mills A, et al. Polymorphisms in the Pseudomonas aeruginosa type III secretion protein, PcrVimplications for anti-PcrV immunotherapy. Microb Pathog. 2010;48(6):197-204.

11. Doring G, Pier GB. Vaccines and immunotherapy against Pseudomonas aeruginosa. Vaccine. 2008;26(8):1011-24.

12. Montor WR, Huang J, Hu Y, Hainsworth E, Lynch S, Kronish JW, et al. Genome-wide study of Pseudomonas aeruginosa outer membrane protein immunogenicity using self-assembling protein microarrays. Infect Immun. 2009:77(11):4877-86

13. Wattam AR, Abraham D, Dalay O, Disz TL, Driscoll T, Gabbard JL, et al. PATRIC, the bacterial bioinformatics database and analysis resource. Nucleic Acids Res. 2014;42(Database issue):D581-91.

14. Tettelin H, Masignani V, Cieslewicz MJ, Donati C, Medini D, Ward NL, et al. Genome analysis of multiple pathogenic isolates of Streptococcus agalactiae: implications for the microbial "pan-genome". Proc Natl Acad Sci U S A. 2005;102(39):13950-5.

15. Tettelin H, Riley D, Cattuto C, Medini D. Comparative genomics: the bacterial pan-genome. Curr Opin Microbiol. 2008;11(5):472-7.

16. Winsor GL, Lam DK, Fleming L, Lo R, Whiteside MD, Yu NY, et al. Pseudomonas Genome Database: improved comparative analysis and population genomics capability for Pseudomonas genomes. Nucleic Acids Res. 2011:39(Database issue):D596-600.

17. Lapierre P, Gogarten JP. Estimating the size of the bacterial pan-genome. Trends Genet. 2009:25(3):107-10.

18. Hilker R, Munder A, Klockgether J, Losada PM, Chouvarine P, Cramer N, et al. Interclonal gradient of virulence in the Pseudomonas aeruginosa pangenome from disease and environment. Environ Microbiol. 2015;17(1):29-46.

19. Valot B, Guyeux C, Rolland JY, Mazouzi K, Bertrand X, Hocquet D. What it takes to be a Pseudomonas aeruginosa? The core genome of the opportunistic pathogen updated. PLoS One. 2015;10(5), e0126468.

20. Ozer EA, Allen JP, Hauser AR. Characterization of the core and accessory genomes of Pseudomonas aeruginosa using bioinformatic tools Spine and AGEnt. BMC Genomics. 2014:15:737.

21. Rasko DA, Rosovitz MJ, Myers GS, Mongodin EF, Fricke WF, Gajer P, et al. The pangenome structure of Escherichia coli: comparative genomic analysis of E. coli commensal and pathogenic isolates. J Bacteriol. 2008;190(20):6881-93.

22. Moriya Y, Itoh M, Okuda S, Yoshizawa AC, Kanehisa M. KAAS: an automatic genome annotation and pathway reconstruction server. Nucleic Acids Res. 2007:35(Web Server issue):W182-5.

23. Backert S, Fronzes R, Waksman G. VirB2 and VirB5 proteins: specialized adhesins in bacterial type-IV secretion systems? Trends Microbiol. 2008;16(9):409-13.

24. Hraiech S, Bregeon F, Rolain JM. Bacteriophage-based therapy in cystic fibrosis-associated Pseudomonas aeruginosa infections: rationale and current status. Drug Des Devel Ther. 2015;9:3653-63. 
25. Ulland TK, Buchan BW, Ketterer MR, Fernandes-Alnemri T, Meyerholz DK, Apicella MA, et al. Cutting edge: mutation of Francisella tularensis mviN leads to increased macrophage absent in melanoma 2 inflammasome activation and a loss of virulence. J Immunol. 2010;185(5):2670-4.

26. Wong HC, Liu SH, Chen MY. Virulence and stress susceptibility of clinical and environmental strains of Vibrio vulnificus isolated from samples from Taiwan and the United States. J Food Prot. 2005;68(12):2533-40.

27. Mosquera-Rendon J, Cardenas-Brito S, Pineda JD, Corredor M, Benitez-Paez A. Evolutionary and sequence-based relationships in bacterial AdoMet-dependent non-coding RNA methyltransferases. BMC Res Notes. 2014;7:440.

28. Benitez-Paez A, Villarroya M, Armengod ME. The Escherichia coli RImN methyltransferase is a dual-specificity enzyme that modifies both rRNA and tRNA and controls translational accuracy. RNA. 2012;18(10):1783-95.

29. Benitez-Paez A, Villarroya M, Armengod ME. Regulation of expression and catalytic activity of Escherichia coli RsmG methyltransferase. RNA. 2012;18(4):795-806.

30. Kimura S, Suzuki T. Fine-tuning of the ribosomal decoding center by conserved methyl-modifications in the Escherichia coli 165 rRNA. Nucleic Acids Res. 2010;38(4):1341-52.

31. Kyuma T, Kimura S, Hanada Y, Suzuki T, Sekimizu K, Kaito C. Ribosomal RNA methyltransferases contribute to Staphylococcus aureus virulence. FEBS J. 2015;282(13):2570-84.

32. Wiehlmann L, Wagner G, Cramer N, Siebert B, Gudowius P, Morales G, et al. Population structure of Pseudomonas aeruginosa. Proc Natl Acad Sci U S A. 2007;104(19):8101-6.

33. Miyoshi-Akiyama T, Kuwahara T, Tada T, Kitao T, Kirikae T. Complete genome sequence of highly multidrug-resistant Pseudomonas aeruginosa NCGM2.S1, a representative strain of a cluster endemic to Japan. J Bacteriol. 2011;193(24):7010.

34. Allewelt M, Coleman FT, Grout M, Priebe GP, Pier GB. Acquisition of expression of the Pseudomonas aeruginosa ExoU cytotoxin leads to increased bacterial virulence in a murine model of acute pneumonia and systemic spread. Infect Immun. 2000;68(7):3998-4004

35. Malathi J, Murugan N, Umashankar V, Bagyalakshmi R, Madhavan HN. Draft genome sequence of multidrug-resistant Pseudomonas aeruginosa Strain VRFPA02, isolated from a septicemic patient in India. Genome Announc. 2013;1(4):e00425-13.

36. Yang Z. Among-site rate variation and its impact on phylogenetic analyses. Trends Ecol Evol. 1996;11(9):367-72

37. Maatallah M, Bakhrouf A, Habeeb MA, Turlej-Rogacka A, Iversen A, Pourcel C, et al. Four genotyping schemes for phylogenetic analysis of Pseudomonas aeruginosa: comparison of their congruence with multi-locus sequence typing. PLoS One. 2013;8(12), e82069.

38. Syrmis MW, Kidd TJ, Moser RJ, Ramsay KA, Gibson KM, Anuj S, et al. A comparison of two informative SNP-based strategies for typing Pseudomonas aeruginosa isolates from patients with cystic fibrosis. BMC Infect Dis. 2014;14:307.

39. D'Auria G, Jimenez-Hernandez N, Peris-Bondia F, Moya A, Latorre A Legionella pneumophila pangenome reveals strain-specific virulence factors. BMC Genomics. 2010;11:181.

40. Kittichotirat W, Bumgarner RE, Asikainen S, Chen C. Identification of the pangenome and its components in 14 distinct Aggregatibacter actinomycetemcomitans strains by comparative genomic analysis. PLoS One. 2011;6(7), e22420.

41. Klockgether J, Cramer N, Wiehlmann L, Davenport CF, Tummler B. Pseudomonas aeruginosa Genomic Structure and Diversity. Front Microbiol. 2011;2:150.

42. Spangenberg C, Heuer T, Burger C, Tummler B. Genetic diversity of flagellins of Pseudomonas aeruginosa. FEBS Lett. 1996;396(2-3):213-7.

43. Winstanley C, Coulson MA, Wepner B, Morgan JA, Hart CA. Flagellin gene and protein variation amongst clinical isolates of Pseudomonas aeruginosa. Microbiology. 1996;142(Pt 8):2145-51.

44. Altschul SF, Gish W, Miller W, Myers EW, Lipman DJ. Basic local alignment search tool. J Mol Biol. 1990;215(3):403-10.

45. Altschul SF, Madden TL, Schaffer AA, Zhang J, Zhang Z, Miller W, et al. Gapped BLAST and PSI-BLAST: a new generation of protein database search programs. Nucleic Acids Res. 1997;25(17):3389-402

46. Edgar RC. MUSCLE: a multiple sequence alignment method with reduced time and space complexity. BMC Bioinformatics. 2004;5:113.

47. Edgar RC. MUSCLE: multiple sequence alignment with high accuracy and high throughput. Nucleic Acids Res. 2004;32(5):1792-7.
48. Korber B. HIV signature and sequence variation analysis. In: Rodrigo A Learn G, editors. Computational analysis of HIV molecular sequences. Dordrecht, Netherlands: Kluwer Academic Publishers; 2000. p. 55-72.

49. Nei M, Gojobori T. Simple methods for estimating the numbers of synonymous and nonsynonymous nucleotide substitutions. Mol Biol Evol. 1986;3(5):418-26.

50. Aoki-Kinoshita KF, Kanehisa M. Gene annotation and pathway mapping in KEGG. Methods Mol Biol. 2007:396:71-91.

51. Letunic I, Doerks T, Bork P. SMART 7: recent updates to the protein domain annotation resource. Nucleic Acids Res. 2012;40(Database issue):D302-5.

52. Finn RD, Bateman A, Clements J, Coggill P, Eberhardt RY, Eddy SR, et al. Pfam: the protein families database. Nucleic Acids Res. 2014;42(Database issue):D222-30.

53. Bardou P, Mariette J, Escudie F, Djemiel C, Klopp C. jvenn: an interactive Venn diagram viewer. BMC Bioinformatics. 2014;15:293.

54. Darriba D, Taboada GL, Doallo R, Posada D. jModelTest 2: more models, new heuristics and parallel computing. Nat Methods. 2012;9(8):772.

55. Letunic I, Bork P. Interactive Tree Of Life V2: online annotation and display of phylogenetic trees made easy. Nucleic Acids Res. 2011;39(Web Server issue):W475-8.

\section{Submit your next manuscript to BioMed Central and we will help you at every step:}

- We accept pre-submission inquiries

- Our selector tool helps you to find the most relevant journal

- We provide round the clock customer support

- Convenient online submission

- Thorough peer review

- Inclusion in PubMed and all major indexing services

- Maximum visibility for your research

Submit your manuscript at www.biomedcentral.com/submit
Biomed Central 